\title{
CORRIGENDUM
}

\section{The Cellular Fatty Acids of the Sulphate-reducing Bacteria, Desulfobacter sp., Desulfobulbus sp. and Desulfovibrio desulfuricans}

By JAMES TAYLOR AND R. JOHN PARKES

Journal of General Microbiology (1983) 129, 3303-3309

Table 1, line 20 of results, beginning ' $16: 1 \omega 5$ ':

under 'Desulfobulbus sp.' the dashes should read ' 1.65 ' (propionate), '11.23' (lactate) and '10.96' $\left(\mathrm{H}_{2} / \mathrm{CO}_{2}\right)$ 